\title{
En torno a la ecología y la fe: EI Excursionismo Religioso y la Sostenibilidad Ambiental en el sur del Perú, anexo de Chapi, Polobaya -Arequipa
}

Around ecology and faith: Religious Hiking and Environmental Sustainability in southern Peruvian, annex of Chapi, Polobaya -Arequipa

\author{
Felipe Mario Zapata Delgado ${ }^{1}$, Ramos Ayme Siomara Jackeline ${ }^{2}$ \\ ${ }^{1}$ Universidad Nacional San Agustín, Arequipa, Perú. \\ ${ }^{2}$ Universidad Cesar Vallejo, Arequipa, Perú.
}

\section{INFORMACIÓN}

Historia del Artículo

Recepción: 13/09/2019

Revisión: 02/10/2019

Aceptación: 15/11/2019

\section{Palabras Clave}

Excursionismo religioso, Chapi, contaminación, sostenibilidad ambiental.

\section{Key Words Religious Hiking, pollution,
environmental sustainability}

\section{DOI}

https://doi.org/10.35286/veritas. v21i1.258

\begin{abstract}
RESUMEN
El propósito es conocer el impacto que existe entre el excursionismo religioso y la sostenibilidad ambiental en el anexo de Chapi, Polobaya. El desplazamiento de las personas hacia los lugares que devienen en lo sacro es un acontecimiento rutinario en el orbe ya que concita atención, encanto y misterio de aquello que se considera, inexplicable y mágico, es decir comprender las peculiaridades del peregrinaje al santuario de Chapi y que es lo que conlleva a que los peregrinos realicen caminatas y actividades ecoturísticas con el fin de llegar al ansiado santuario; y de otro lado observar que si se ha ponderado los riesgos de estos traslados respecto del marco ambiental, es decir si existe una propuesta ecológica para comprender la capacidad de carga de la zona de Chapi. La metodología es mixta, se privilegia los testimonios de los viajeros y comerciantes mediante una encuesta que tuvo una muestra de 277 visitantes a la festividad de Chapi, en temporada alta. Los resultados se evidencian en que un $62 \%$ de visitantes consideran que el nivel de desarrollo del excursionismo religioso en Chapi es regular y un 37\% considera inadecuado, lo que indica que la actividad religiosa no está desarrollando de una manera ambientalmente apropiada; en cuanto a sostenibilidad ambiental un $63 \%$ de visitantes, consideran malo, y un $39 \%$ indica que es regular, por lo que se requiere una adecuada gestión ambiental en zona comercial, fuentes de agua y santuario.
\end{abstract}

\begin{abstract}
The purpose is to know the impact that exists between religious hiking and environmental sustainability in the annex of Chapi, Polobaya. The displacement of people to the places that become sacred is a routine event in the world as it attracts attention, charm and mystery of what is considered inexplicable and magical, that is, to understand the peculiarities of the pilgrimage to the sanctuary of Chapi and which is what leads the pilgrims to make walks and ecotourism activities in order to reach the coveted sanctuary; and on the other hand, note that if the risks of these transfers have been weighed against the environmental framework, that is, if there is an ecological proposal to understand the carrying capacity of the Chapi area. The methodology is mixed, the testimonies of travelers and merchants are privileged through a survey that had a sample of 277 visitors to the Chapi festival, in high season. The results are evidenced in that $62 \%$ of visitors consider that the level of development of religious hiking in Chapi is regular and $37 \%$ consider it inappropriate, which indicates that religious activity is not developing in an environmentally appropriate way; In terms of environmental sustainability, $63 \%$ of visitors consider it bad, and $39 \%$ indicate that it is regular, which is why adequate environmental management is required in the commercial area, water sources and sanctuary.
\end{abstract}

\section{INTRODUCCIÓN}

Existe un prurito a lo insondable en el ser humano, donde ante lo inexplicable y que la rareza del peregrino encuentra en cada acto, uso, forma o condición; adscribir valoraciones sobrenaturales cargados de fe y devoción, es el caso del santuario de Chapi. La práctica de llegar este lugar a definido formas básicamente el de hacer una caminata desde la ciudad de Arequipa hasta el santuario pero estas experiencias en la actualidad generan pingues ganancias por las diversas formas de comercio que existen entendiendo que "el traslado de viajeros-peregrinos contribuye al desarrollo económico de

Correspondencia: mzapata1@unsa.edu.pe un país, y los actores que participan en este sector obtienen ventajas económicas como cualquier otra empresa" (Juárez et al, 2010, p.46); los que se benefician fundamentalmente son las empresas que se dedican a la hotelería y promoción del turismo; sin embargo, no todos ganan y los que pierden son los más pobres (Bonilla, 2012); además del impacto ambiental que generan.

El Perú al ser heredera de la cristiandad esta inherente a este fervor la cual ha cultivado festividades religiosas como del señor de los Milagros (Lima), el Cristo Cautivo de Ayabaca (Piura), el señor de Muruhuay (Junín) entre otros, lamentablemente a una falta de políticas públicas adecuadas hay una conciencia ambiental precaria origina una degradación en el contexto de peregrinación provocando daños irreversibles

Arequipa no escapa de esta religiosidad y se denota 
con una pleitesía a la virgen Maria conocida mejor como la Candelaria la cual se festeja con "marcada devoción en los santuarios principalmente de Cayma, Characato y Chapi, han hecho que sea bautizada como, la Roma del Perú" (Málaga, 2001, p.4). Esta virgen cuya advocación mariana antigua, tiene una imagen en el Santuario Chapi, y es considerada la patrona de Arequipa (Resolución Ministerial Nº 048, 2012).

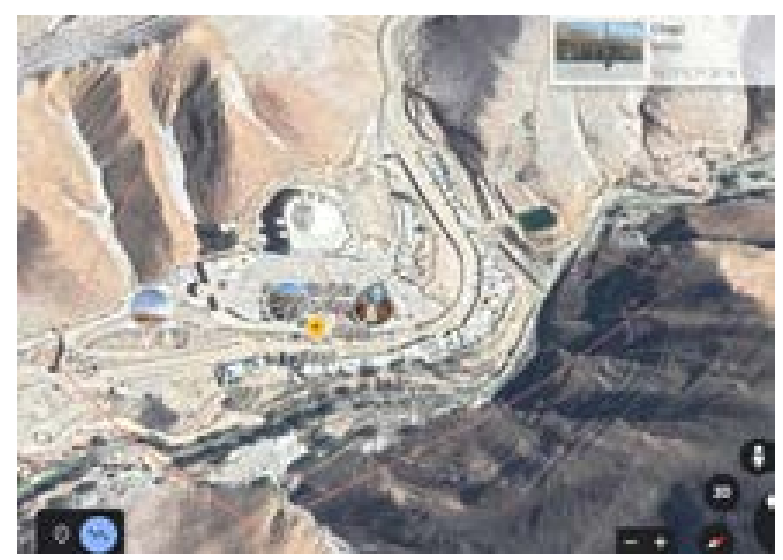

Fig. 1: Santuario de Chapi; Fuente google Earth

Dichas peregrinaciones religiosas junto con todas sus connotaciones e implicancias generan un impacto en la capacidad de carga, principalmente en temporadas altas (fiestas importantes), problemas ambientales contaminándose el agua y suelo, incremento de tráfico de vehículos, afectando de una forma importante al paisaje y la alteración del medio ambiente.

Este trabajo se realizó en el anexo de Chapi, distrito de Polobaya a $90 \mathrm{~km}$ de la ciudad de Arequipa, presenta un clima seco, característico del matorral desértico húmedo. Etimológicamente "chapi" proviene del aymara que significa "espino" o "cactus" con el que probablemente se nombró a esa quebrada debido a la abundancia y variedad de aquella planta en el valle" (Clavell, 1999, p.15). Es en este pueblo donde se encuentra la virgen de la Candelaria de Chapi, la cual genera un gran desplazamiento de excursionistas religiosos (peregrinos), "su fiesta se celebra el 2 de febrero, día de la Purificación o Candelaria, pero los peregrinos han escogido de preferencia el $1^{\circ}$ de mayo, en que se da inicio al mes dedicado a María, y también el 8 de setiembre, fiesta de su Natividad...en esas fechas, especialmente, se puede apreciar una concentración bastante significativa, un verdadero mar humano" (Vargas, 1956, 152). A la par de esta peregrinación existe un impacto sobre el ambiente donde la degradación ambiental, es por lo que nos hemos planteado las siguientes preguntas de investigación ¿cuál es la relación entre el excursionismo religioso y la sostenibilidad ambiental en el anexo de Chapi, Polobaya - 2018? ¿Cuál es el nivel de desarrollo del excursionismo religioso en Chapi? ¿Cuál es el grado de sostenibilidad ambiental en Chapi? ¿Cuál es la capacidad de carga del Santuario de Chapi?,

\section{Peregrinación, excursionismo y fe}

\section{a) Peregrinación}

En latín peregrinus, designaba al individuo que viajaba por diversos países o aquellos que no eran reconocidos como ciudadanos, el vocablo peregrinatio significa viaje, visita a países, según Rebic es la práctica religiosa de visitar lugares sagrados (2000)

Los centros mas concurridos desde la óptica cristiana son el Vaticano, Lourdes, Fátima, básicamente en términos macros definen el agradecimiento por una promesa cumplida un milagro o simplemente la fe, y estas formas son llevadas a prácticas votivas en sus lugares de origen como pasacalles, desfiles o fiestas patronales.

Las peregrinaciones representan flujos de masas de personas que se desplazan de sus lugares hacia estos centros posibilitando mejoras en las economías de los territorios que son visitados, lo cual beneficia el desarrollo de la población involucrada en este negocio, pero no siempre se toma en cuenta el orbe ambiental.

"por un lado posibilita una derrama económica que beneficia a los habitantes del sitio visitado al generar inversión en infraestructura y servicios diversos, promoviendo la creación de empleo y el bienestar económico, por otro lado, tiene efectos no siempre benéficos sobre el entorno natural, el patrimonio físico y cultural del lugar visitado principalmente debido a que los sitios de fe no se desarrollan bajo una planificación urbana que facilite el desarrollo de la actividad turística religiosa, sino que su crecimiento se realiza buscando resolver prioritariamente necesidades de corto plazo" (Martinez,2013,p.2),

Según Martínez (2012) el viaje por motivo religioso, es la determinación que lleva la fe intrínseca a un desplazamiento de un lugar de origen hacia otro considerado como sagrado, pero lo peculiar radica en tener o estar en espacio con la divinidad que no puede ser otro sitio apreciado entendido como profano.

\section{b) Excursionismo}

González (2008) define el excursionismo religioso, como "el desplazamiento de visitantes hacia un centro, un lugar geográfico que las personas entienden como lugar santo, lugar de encuentro con Dios, no permaneciendo más de 24 horas" (p, 12.), que se diferencia del turismo religioso por la permanencia, según Morán (2011) "son los viajes tradicionales a un santuario, orientados, guiados y regulados por organizaciones religiosas u operadores turísticos, para así difundir la historia y trascendencia de cada lugar visitado, pernoctando en el lugar" ( $p, 13$.)

Parellada afirma que "el elemento clave para definir el turismo religioso es la motivación religiosa.... la motivación religiosa coexiste con la curiosidad o el interés cultural por ciertas formas de entender el mundo, diferentes a la propia" (2009). La Organización Mundial del Turismo (1998) menciona que "el excursionismo, es el que no usa alojamiento colectivo o privado del país visitado, llamado visitas o excursiones del día y como una las motivaciones de viaje mencionan, Religión / peregrinaciones" (p. 6).

Las peregrinaciones a Chapi, no son guiadas ni reguladas por alguna organización religiosa y la permanencia no es más de 24 horas, en la mayoría de casos, principalmente porque Chapi no ofrece la infraestructura adecuada (hoteles) 
para pernoctar, por lo que el término adecuado en ésta investigación es excursionismo religioso.

Ya sea peregrinaciones, excursionismo o turismo religioso, mueven grandes masas de personas, trayendo consigo beneficios económicos. Así lo menciona Germain (2007) "muchas agencias promocionan viajes que incluyen paquetes y programas para los peregrinos a lugares santos, y es indudable que el fenómeno del peregrinaje en el mundo está prosperando, debido a la connotación económica y social, que tiene el turismo religioso, observado sobre todo en el orbe cristiano occidental" ( p.21).

\section{La fe ¿Impacto en el ambiente?}

La literatura sobre la fe es enorme como la religiosidad y lo sobrenatural que envuelve al hombre que busca respuestas y solo con el uso de un razonamiento lógico no puede encontrar, para Para Francisco Díaz "fe es una virtud sobrenatural que capacita al hombre a asentir firmemente a todo lo que Dios ha revelado, no por su evidencia intrínseca sino por la autoridad de Dios que revela. La fe es ante todo adhesión personal del hombre a Dios; es al mismo tiempo e inseparablemente el asentimiento libre a toda la verdad que Dios ha revelado» (VATICANO).

Pero es humano ¿que la fe de sus practicantes lleve a impactar el ambiente en aras de su religiosidad? tratemos de adentrarnos en esta perspectiva, ya que es probable que la fe como motivación sea el centro de la problemática, pero que en su nombre se realice actividades dañinas que el visitante no reconoce sino más bien soslaya, entonces es necesario la disquisición respectiva.

\section{a) Enfoques y puntos de vista.}

Alcántara (2011) en la Guía de Práctica Sanitaria, las peregrinaciones pueden generar riesgos a la salud entre los peregrinos y a las poblaciones ubicadas en el trayecto, como contraer infecciones gastrointestinales, insolación, deshidratación, cansancio extremo o complicaciones de enfermedades que previamente padece el peregrino.

En el Perú los estudios de impacto de los viajes o turismo en zonas naturales, son muy escasos Tinoco (2003) menciona algunos impactos del turismo, por ejemplo; en Lima, en el verano produce un congestionamiento automovilístico por traslado a las playas, asimismo en Semana Santa, Ayacucho, es "tomada por asalto" por caravanas de vehículos provenientes de la capital. Ni que decir de la ciudad del Cuzco.

El Gobierno Regional de Puno, menciona que en la fiesta de la candelaria en el año 2014 se recogieron más de 30 mil toneladas de basura, a pesar de que orientan a los turistas; alcanzando bolsas para los residuos y no usen residuos como: platos descartables, bolsas de plástico y otros, Sin embargo es difícil evitar la contaminación del suelo y atmosférica.

\section{b) Sostenibilidad: necesidad en las peregrinaciones.}

En el informe "Nuestro Futuro Común" se formaliza el concepto de sostenibilidad el cual busca "satisfacer las necesidades de las generaciones presentes sin comprometer el derecho de las generaciones futuras al satisfacer sus propias necesidades" (Jankilevich, 2003, p.8). Según Constanza (1994, Citado en Marbán, 2006, p. 33 ) “....existen tres políticas para conseguir la sostenibilidad; la primera es conseguir una tasa sobre la destrucción del capital natural con el fin de reducir o eliminar la destrucción del mismo, la segunda es aplicar el principio contaminador-pagador aplicado a productos contaminadores con el fin de incentivar a los productores a mejorar el entorno y, por último, un sistema de aranceles ecológicos que permita a los países aplicar las dos políticas anteriores sin forzar a sus productores a moverse a otros lugares para conseguir mantener la competitividad".

La sostenibilidad busca hacer del presente un hogar para nuestros hijos ya que a ellos pertenece el futuro, para Gallopín (2003) el prerequisito ético de la sostenibilidad, es la preservación del ambiente bajo un sistema socio ecológico, que quiere decir un sistema formado por un componente social en dinámica con un componente ecológico.

La actividad turística-religiosa, la sostenibilidad debe entenderse como planificación es la administración de recursos que permitan satisfacer las necesidades de los turistas y la ciudad destino y conservarlos para el futuro. (Martínez,2003)

La dimensión turística del desarrollo para por el conocimiento sistémico entre el ambiente y la sociedad la cual son subyacentes a el logro de la libertad y competencias humanas

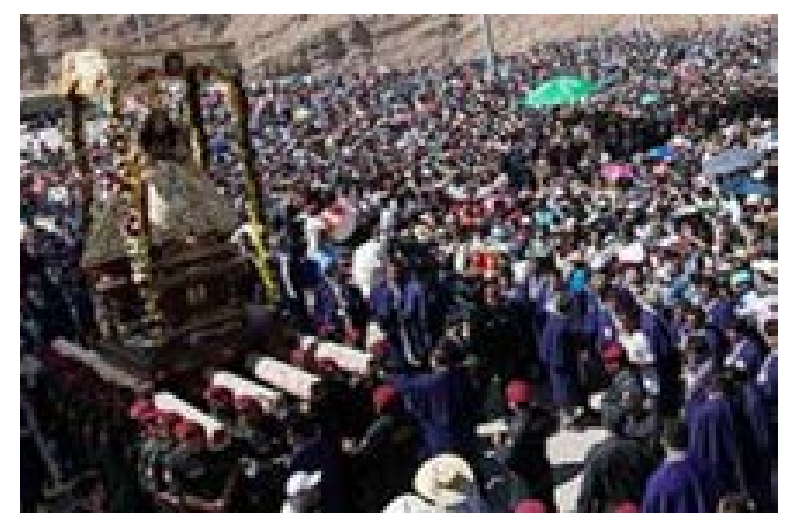

Fig. 2: Peregrinos y capacidad de carga del santuario, cortesía el Comercio

\section{c) Capacidad de carga}

Tratar este aspecto resulta fundamental para conocer cuánto es el impacto sobre la zona o área de peregrinación; Blanco \& Benayas (1998) mencionan que la capacidad de carga, está relacionada con "la sensación de agobio que tienen las personas que visitan un determinado lugar, al encontrarse con un número elevado de visitantes. A partir de un cierto nivel de masificación, la experiencia recreativa del usuario tiende a valorarse como negativa, independientemente de las virtudes escénicas o naturales del lugar visitado".

\section{Métodos y fuentes}

Investigación descriptiva correlacional cuya muestra fue 277 visitantes al anexo de Chapi, se aplicó una prueba retest a 10 visitantes, la técnica que se utilizo fue la encuesta con su cuestionario basado en el formulario Likert, el cual sirvió para valorar las opiniones de los peregrinos y además la percepción que tuvieron de los comercios y del uso indiscriminado del espacio y los recursos que conlleva la visita al santuario, Además se utilizó la correlación de Pearson. 


\section{RESULTADOS}

La virgen de Chapi entre la devoción y el esparcimiento

Como señalamos líneas arriba son dos aspectos fundamentales que marcamos en nuestra investigación: El excursionismo religioso hacia el santuario de Chapí e impacto ambiental. El excursionismo religioso presenta algunas características que hemos evaluado: la permanencia en Chapí, la motivación que lleva al peregrino a la realización de este viaje, la responsabilidad ambiental del viajero y la capacidad de carga; Impacto ambiental, la mirada es desde la zona comercial, Fuentes de agua y propiamente el santuario.

\section{El excursionismo religioso hacia el santuario de Chapí}

La ruta que realizan atraviesan los distritos de Paucarpata, Sabandía, Characato hasta llegar a Yarabamba, el pueblo de Sogay. la "Cuesta de Hornillos (Gonzales, Chong, \& al)

Existe varias formas de iniciar esta excursión generalmente cuando son familias completas o situaciones en que impera pedimentos cruzados con la fe; personas de la tercera edad, además de otras motivaciones como el de hacer el viaje rápido, se toman los transportes que se encuentran en la explanada Andrés Avelino Cáceres. Mientras que muchos peregrinos prefieren hacer la caminata desde Arequipa hasta el santuario, finalmente en su día central se abarrotan poblaciones de excursionistas muy grandes en la zona de Chapi. Pero ¿qué es lo que determina su permanencia, la mayoría tiene una presencia real en el santuario de un día o menos y luego se retorna Arequipa o Moquegua?, entonces la motivación que llevan a estos peregrinos para realizar este excursionismo de acuerdo al estudio, muestra que el 59\% de los visitantes encuestados manifiestan que su permanencia es menor a un día y un $22 \%$ manifiesta permanecer más lo cual sugiere que entre los peregrinos, excursionistas o turistas que vistan Chapi su permanencia es corta, debiendo tomarse en cuenta el traslado por la ruta definida sea a pie o en vehículo hace que el santuario tenga una motivación diferente al solo vista normal a una candelaria como sucede en las iglesias de Arequipa, incluso se nota que hay varias homónimas de lo que se conoce en como Chapi Grande, vemos en la zona de Cayma denominada Charcani, Chapi Chico, y muchos lugares que han redefinido nuevas formas de fe o religiosidad sobre esta virgen, pero Chapi Grande a pesar de esta contradicción en la permanencia el traslado de los visitantes es mayor. Otro aspecto es la motivación que lleva al peregrino a la realización de este viaje, se cree que el fundamento es la fe lo cual aparentemente se evidencia por traslado masivo, pero de los resultados obtenidos tenemos que el $56.7 \%$ de visitantes a Chapi, consideran que tienen otra motivación para viajar a Chapi, un sector mayoritario asume la posibilidad de "milagro" es decir, la casa propia, el vehículo, la enfermedad, el retorno de la pareja infiel, es decir problemas personales o proyectos de vida, lo cual indica que se puede considerar viajar a Chapi por un motivo distinto al religioso y que en el lugar regularmente se realiza otras actividades distintas a la fé y religiosidad. Y solo un $30.7 \%$ consideran que la motivación de viaje es participar de la celebración religiosa en Chapi. La responsabilidad ambiental del viajero, tenemos ante la fragilidad de este ambiente el $49 \%$ de visitantes encuestados manifiestan estar en desacuerdo que la actividad religiosa se realice de una manera ambientalmente responsable, y si se suma los porcentajes correspondientes a los niveles muy en desacuerdo y en desacuerdo se puede apreciar una cifra de $89 \%$, lo que indica que el anexo de Chapi carece de buenas prácticas ambientales, lo cual es peligroso, ya que los visitantes no asumen el mantenimiento del ambiente provocando un impacto su presencia en el santuario muy perjudicial. Respecto de la capacidad de carga; se obtuvo que un $83.4 \%$ de visitantes encuestados consideran el nivel de capacidad de carga inadecuado, lo que quiere decir que el número de visitantes en Chapi, no es el adecuado y que está recibiendo viajeros más de lo permitido. Este efecto es observado ya que tenemos una sobrecarga con visitantes, negocios de expendio de comidas, souvenirs, elementos de parafernalia y sobre todo la acumulación de vehículos de transporte como particulares, rompiendo las formas adecuadas del sistema en el santuario, lo que indica que la actividad religiosa requiere una planificación y regular la capacidad de viajeros que puede recibir el anexo de Chapi.

\section{a) Impacto ambiental.}

Tenemos que la percepción de los visitantes sobre el impacto que se genera en un $62 \%$ consideran el desarrollo del excursionismo religioso en un nivel regular y un $37 \%$ en un nivel inadecuado, tan es así que la zona comercial, es decir el espacio para transporte y expendio de productos que se han distribuido para las diversas actividades son inadecuados, $65.7 \%$ de visitantes encuestados, manifiestan estar muy en desacuerdo, con respecto al estacionamiento transporte terrestre alrededor del santuario. Fuentes de agua, teniendo la creencia que los manantes son benditos y tienen atributos excepcionales, además de ser utilizado por los comerciantes para la preparación de los alimentos; se ha generado un impacto agresivo en estos espacios; el $63 \%$ de visitantes encuestados consideran la sostenibilidad ambiental en las áreas del río en un nivel malo, seguido de un $31 \%$ que consideran un nivel regular, concluyendo que las zonas del río carecen de un uso responsable y sostenible por parte de los visitantes y comerciantes, aunando a esto la falta de un mantenimiento adecuado y oportuno de los alrededores del río. El santuario. Estamos en el entendimiento que el santuario y sus actores, - sacerdote, sacristán, encargados de la municipalidad, del arzobispado, trabajadores- que deben inducir y sensibilizar en las buenas practicas, cuidado, protección y mantenimiento, un $43 \%$ de visitantes encuestados manifiestan que la parroquia no educa a los visitantes, frente a un $23 \%$ que está de acuerdo, que la parroquia advierte a los visitantes sobre el cuidado y preservación del santuario. Entonces se puede decir, que la parroquia intenta educar a los peregrinos, pero aún son pocos sus esfuerzos. A esto se suma que la contaminación en Chapi, por el desarrollo de la actividad religiosa, un $48 \%$ de visitantes están de acuerdo que el fin religioso como actividad contribuye a la contaminación en el anexo de Chapi, ya que es la única actividad económica (transporte, comida, recursos y elementos para la parafernalia) en este lugar. Un 63\% de visitantes encuestados, consideran que el nivel de sostenibilidad ambiental en el anexo de Chapi es malo, un $39 \%$ indica que es regular, por lo que se puede indicar que se requiere una adecuada gestión ambiental en zona comercial, fuentes de agua y santuario. La correlación entre excursionismo religioso y sostenibilidad ambiental, se obtuvo un coeficiente de 0,123 y un nivel de significancia de 0.04 , lo cual indica que existe una correlación baja 


\begin{tabular}{cccc}
\hline & & $\begin{array}{c}\text { Excursionismo } \\
\text { religioso }\end{array}$ & $\begin{array}{c}\text { Sostenibilidad } \\
\text { ambiental }\end{array}$ \\
\hline $\begin{array}{c}\text { Excursionismo } \\
\text { religioso }\end{array}$ & $\begin{array}{c}\text { Correlación } \\
\text { de Pearson }\end{array}$ & 1 &, $123^{*}$ \\
& Sig. (bilateral) & &, 041 \\
& $\mathrm{~N}$ & 277 & 277 \\
$\begin{array}{c}\text { Correlación } \\
\text { de Pearson }\end{array}$ &, $123^{*}$ & 1 \\
ambienibilidad & Sig. (bilateral) &, 041 & \\
& $\mathrm{~N}$ & 277 & 277 \\
\hline
\end{tabular}

*. La correlación es significante al nivel 0,05 (bilateral).

\section{CONCLUSIONES}

La relación entre el excursionismo religioso y la sostenibilidad ambiental en el anexo de Chapi, Polobaya - 2018, es mínima en la medida en que el excursionismo religioso provoca impacto ambiental muy fuerte asumiendo que la relación es más bien baja

El nivel de desarrollo del excursionismo religioso en Chapi es muy alto la cual se lleva a cabo no siempre debido a la fe es decir, donde se participe en los actos litúrgicos y el acompañamiento en la devoción mariana, ya que en la mayoría de los casos los visitantes lo hacen por situaciones generalmente para realizar alguna petición; y otro gran grupo señala que justamente la religiosidad representa algún ingreso económico en su provecho. Existe una razón poderosa para que los visitantes se trasladen al santuario es el hecho de realizar el viaje, salir de Arequipa, encontrarse con la naturaleza y sus propias motivaciones de vida, como esparcimiento, peticiones, libertad. No sucede lo mismo con la virgen de Chapi del cercado de Arequipa, a pesar que en la fiesta patronal se atiborran estos lugares, no hay punto comparación con los feligreses que se trasladan hacia el santuario.

El grado de sostenibilidad ambiental en Chapi, lamentablemente al ser el santuario un factor de peregrinaje los visitantes argumentan que al no tener buenas prácticas y existir mínimamente información sobre el mantenimiento del lugar es que la sostenibilidad es mínima, ya que las acciones de la municipalidad y los diversos actores en la zona del santuario no son contundentes por lo que el espacio está seriamente impactado por el flujo.

La capacidad de carga del Santuario de Chapi, en temporada alta rebasa las posibilidad de un uso racional de los recursos ya que son los visitantes, el transporte masivo, los expendios de comida y de souvenirs, entre otros, además de la distribución incorrecta de las zonas de parqueo y del paso peatonal, observándose una capacidad de carga muy alta provocando daños al medio ambiente; pero que durante el año la capacidad de carga es regular.

\section{REFERENCIAS BIBLIOGRÁFICAS}

1. Acerenza, A. (2000). Administración del Turismo. Conceptualización y Organización. Volumen 1. México: Editorial Trilla.

2. Alcántara, J. R. (2011) Guía de Práctica Sanitaria No. 4. Peregrinaciones. Instituto de Salud del Estado de México / Coordinación de Regulación Sanitaria

3. Álvarez, R \& Espinoza, G. (2008). Turismo y desarrollo local. Proyecto para convertir "Cocodrilo" en pueblo turístico sostenible en un área protegida de la Isla de la Juventud. Cuadernos de Turismo, n²2. Pp. 9-33. Cuba

4. Alzate, B.E. (2008). Diagnóstico de la sostenibilidad ambiental. Colombia: Instituto de Estudios Ambientales - IDEA.

5. Andrade, A. (2004). Lineamientos para la aplicación del enfoque ecosistémico a la gestión integral del recurso hídrico. Serie Manuales de Educación y Capacitación Ambiental Vol. VIII. México: PNUMA.

6. Blanco, R. \& Benayas, J. (1998). Los estudios de capacidad de acogida y su contribución para establecer modelos de turismo sostenible en espacios naturales. España: Universidad Autónoma de Madrid. DE.

7. Bonilla, L. (2012). Los peregrinos. Sus orígenes, rutas y religiones. España: Editorial Biblioteca Nueva.

8. Briceño, Elsa (2010) Diagnóstico de los impactos ambientales generados por la actividad turística en la localidad de Río Negro. (Tesis) Venezuela. Instituto Universitario Tecnología del Estado Trujillo. Recuperado de: http:/www.monografias. com/trabajos99/diagnostico-impactos-ambientalesgenerados-actividad-turistica-localidad-rio-negro/ shtml\#ixzz3A6XP7iJ8. (21.Nov.2014).

9. Cazes, 0. (1992): Fundamentos de la capacidad geográfica turística. España: Rosny.

10. Cànoves, G. \& Blanco, A. (2011). Turismo religioso en España: ¿La gallina de los huevos de oro? Una vieja tradición, versus un turismo Emergente. Cuadernos de Turismo, $\mathrm{n}^{\circ}$ 27. pp. 115-131 España: Universidad de Murcia.

11. Clavell, A. (1999). Breve historia del Santuario de Chapi. Arequipa, Perú: Fuentes impresiones.

12. Echamendi, P.(2001). La capacidad de carga turística. Aspectos conceptuales y normas de aplicación. MANUALES de Geografla de la Universidad complutense. pp. 11-30. ISSN: 0211-9803

13. Eslava, R. (2002). Valoración Económica de los pasivos Ambiéntales Generados por las Empresas Hoteleras de la Cuenca del Río Mucujún: Técnica del Gasto Preventivo. (Tesis)Venezuela: Universidad de Los Andes.

14. Gallopín, G. (2003). Sostenibilidad y desarrollo sostenible. Un enfoque sistémico. CEPAL. Chile

15. Germain, A. (2007). El mercado español de turismo religioso, un sector prometedor para la profesión. Revista EDITUR, No 2480, p. 21. España

16. Gligo, N. (2001). La Dimensión Ambiental en el Desarrollo de América Latina. Comisión Económica para América Latina y el Caribe (CEPAL). Chile.

17. González, J. C. (2008). Historia de la Basílica de San Juan de los Lagos. Revista El turismo religioso. Sus perfiles. pp.1-5. México

18. Hernández, R., Fernández, C. \& Baptista, P. (2006). 
Metodología de la investigación. Colombia. (4 ${ }^{\mathrm{a}}$ ed.) México: Mc Graw-Hill.

19. Huamaní, W. (1998). Diagnóstico Ambiental Preliminar del Parque Nacional Huascarán. (Tesis). Perú. UNALM.

20. Jankilevich, Silvia (2003). Las cumbres mundiales sobre el ambiente. Estocolmo, Río y Johannesburgo. 30 años de Historia Ambiental. Documento de Trabajo $\mathrm{N}^{\circ}$ 106, Universidad de Belgrano. Argentina. Recuperado de: http://www.ub.edu.ar/investigaciones/ dt_nuevos/106_jankilevich.pdf.

21. Luque, T.(2000). Técnicas de análisis de datos de investigación. Perú. Ediciones Pirámide.

22. "La carta del turismo sostenible" (1995). España. Recuperado de www.omt.org.

23. Marbán, R. (2006). Impulsora del desarrollo sostenible y de la protección del medio ambiente en Europa y España. Boletín Económico de ICE No 2899. España

24. Martínez, A. (2003). El turismo sostenible: herramienta para la lucha contra la pobreza. Perú.

25. Martínez, R. (2013). Santuarios, fiestas patronales, peregrinaciones y turismo religioso. México: Fundación Universitaria Andaluza Inca Garcilaso para eumed. net. Recuperado de:http://www.eumed.net/librosgratis/2013/1281/index.htm (07.SET 2014).

26. Martínez, R. (2012). Desarrollo regional cimentado en el turismo religioso. México: Estudios Organizacionales del Centro Universitario de los Altos de Jalisco

27. Molina, S. (2004). Turismo y Ecología. México: Editorial Trillas.

28. MORÁN, V. (2011). Puesta en valor del intangible de la fiesta de la virgen de la caridad de la ciudad de Mira. (Tesis). Ecuador: Pontificia Universidad Católica del Ecuador.

29. Morillo, M. (2002). Sustentabilidad socio-ambiental de la actividad turística receptora. Reflexiones y alternativas Revista Economía No. 18. Pp. 69-107. Venezuela.

30. Organización Mundial del Turismo. (1998). Introducción al Turismo OMT. España. Recuperado de: www.omt.org.pe (16.DIC.2014)

31. Parellada, J. E. (2009). El turismo religioso. Sus perfiles. Jornadas de Delegados de Pastoral de Turismo, Ávila. Conferencia Episcopal Española: 1-27. España

32. Pearce, D. \& Turner, R. (1995). Economía de los Recursos Naturales y del Medio Ambiente. España Celeste Ediciones.

33. Rabinovich, J. \& Torres, F. (2004). Caracterización de los síndromes de sostenibilidad del desarrollo. El caso de Argentina. Proyecto "Evaluación de la sostenibilidad en América Latina y el Caribe" (ESALC) División de Desarrollo Sostenible y Asentamientos Humanos. Argentina: Colsem.

34. Robles, J. (2001) Turismo religioso. Alternativa de apoyo a la preservación del patrimonio y desarrollo. España: Biblio 3w.

35. SECTUR. (2009). Dimensionamiento del Turismo espiritual en México. Universidad de Guadalajara, Tepatitlán de Morelos, Jalisco. Recuperado de: http://www.sectur. gob.mx/work/models/sectur/Resource/15653/ IMENSIONAMIENTODELTURISMOESPIRITUAL. pdf (11.AGO.2014)
36. Shepherd, Gill. (2006). El Enfoque Ecosistémico: Cinco pasos para su implementación. Suiza y Reino Unido: Unión Mundial para la Naturaleza-UICN.

37. Tinoco, O. (2003). Los impactos del turismo en el Perú vol. 6. Perú: Industrial data

38. Unchupaico, Ángel D. (2010) "Gestión ambiental y tratamiento de residuos urbanos: propuesta para el distrito de El Tambo, a partir de experiencias exitosas" (Tesis) Perú. Universidad Nacional del Centro del Perú. Recuperado de:http://es.slideshare.net/fredyvalencia/ plan-de-tesis-4902975 (10. Oct. 2014)

39. Vargas, R. \& Ugarte S. J. (1956). Historia del Culto de María en Iberoamérica y de sus Imágenes y Santuarios más celebrados, $3^{\mathrm{a}}$ edición, Tomo II, p. 152. España.

40. Vasconi, M. (2002). El impacto geográfico del excursionismo Religioso (católico-mariano) en Luján -Provincia de Buenos Aires 2002 (Tesis) Argentina. Universidad del Salvador. Recuperado de: http:// www.amazon.es/Impacto-Geografico-ExcursionismoReligioso-Lujan/dp/384736440507-. (07. Oct. 2014)

41. Vega, L. (2005). Hacia la sostenibilidad ambiental del desarrollo. Construcción de pensamiento ambiental práctico a través de una política y gestión ambiental sistémica. Ecoediciones

42. Viceministro de Patrimonio Cultural e Industrias culturales. Resolución ministerial No 048. (2012).Perú. Recuperado de: www.mincetur.gob.pe

43. Cristiano, M. r. (2013). Las peregrinaciones. Pistas para el dialogo.

44. Gonzales, Chong, \& al, e. (s.f.). Virgen de Chapi. Obtenido de : https://es.wikipedia.org/wiki/Virgen_de_ Chapi?oldid $=92442462$

45. Rebic, 1. d.-A. (2000). Documento de la Santa Sede sobre el Peregrinaje. Roma: Neum.

46. VATICANO, C. (s.f.). TEMA 3. LA FE SOBRENATURAL. Obtenido de http://multimedia. opusdei.org/pdf/es/3.pdf 